\title{
Disturbance and recovery: a synthesis of microbial community reassembly following disturbance across realms
}

\author{
Stephanie Jurburg${ }^{1}$, Shane Blowes ${ }^{1}$, Ashley Shade $^{2}$, Nico Eisenhauer ${ }^{3}$, and Jonathan \\ Chase $^{1}$ \\ ${ }^{1}$ German Centre for Integrative Biodiversity Research (iDiv) Halle-Jena-Leipzig \\ ${ }^{2}$ Michigan State University \\ ${ }^{3}$ University of Leipzig
}

November 29, 2021

\begin{abstract}
Disturbances alter the diversity and composition of microbial communities, but whether microbiomes from different environments exhibit similar degrees of resistance or rates of recovery has not been evaluated. Here, we synthesized 86 time series of disturbed mammalian, aquatic, and soil microbiomes to examine how the recovery of microbial richness and community composition differed after disturbance. We found no general patterns in compositional variance (i.e., dispersion) in any microbiomes over time. Only mammalian microbiomes consistently exhibited decreases in richness following disturbance. Importantly, they tended to recover this richness, but not their composition, over time. In contrast, aquatic microbiomes tended to diverge from their pre-disturbance composition following disturbance. By synthesizing microbiome responses across environments, our study aids in the reconciliation of disparate microbial community assembly frameworks, and highlights the role of the environment in microbial community reassembly following disturbance.
\end{abstract}

Disturbance and recovery: a synthesis of microbial community reassembly following disturbance across realms

Authors : Stephanie D. Jurburg*1,2, Shane A. Blowes ${ }^{1,3}$, Ashley Shade ${ }^{4}$, Nico Eisenhauer ${ }^{1,2}$, Jonathan M. Chase ${ }^{1,3}$

${ }^{1}$ German Centre for Integrative Biodiversity Research (iDiv) Halle-Jena-Leipzig

${ }^{2}$ Institute of Biology, Leipzig University

${ }^{3}$ Institute of Computer Science, Martin-Luther University Halle-Wittenberg, Halle (Saale)

${ }^{4}$ Department of Microbiology and Molecular Genetics, Department of Plant Soil and Microbial Sciences, and Program in Ecology, Evolution and Behavior, Michigan State University, East Lansing MI 48824

*Correspondence: Stephanie D. Jurburg, s.d.jurburg@gmail.com;German Centre for Integrative Biodiversity Research (iDiv) Halle-Jena-Leipzig, Puschstraße 4, 04103, Leipzig, Germany

Email addresses: S.A.B. (shane.blowes@idiv.de); A.S. (shadeash@msu.edu) N.E. (nico.eisenhauer@idiv.de); J.M.C. (jonathan.chase@idiv.de)

Keywords : community assembly, microbiome, bacteria

Running title : Community reassembly in microbiomes

Article type : Letter 


\begin{abstract}
: 138 words
Main text: 3,804 words

Figures : 6

References : 85

Author statement : S.D.J., A.S., N.E., and J.M.C conceived of the idea; S.D.J. obtained the data, performed bioinformatics and null models, and wrote the first draft; S.A.B. performed statistical analyses; all authors contributed to revisions.

Data statement: Accession numbers for the datasets included in this study are included in the supplementary data of this article, and are publicly available. Code used for sequence processing and null modelling is publicly available athttps://github.com/drcarrot/DisturbanceSynthesis, and code used for statistical analyses is publicly available athttps://github.com/sablowes/microbiome-disturbance. Both repositories will be combined and archived at Zenodo upon acceptance.
\end{abstract}

Novelty statement: We compare the recovery of disturbed microbial communities across 86 aquatic, mammalian, and soil time series by measuring richness, compositional dispersion, and compositional turnover over time. We find that patterns of community reassembly in microbiomes are environment-specific, reconciling existing, environment-specific hypotheses of microbiome responses to disturbance. This is the first study to explicitly compare microbial community reassembly across environments within time scales that are relevant to bacterial life histories.

\title{
ABSTRACT
}

Disturbances alter the diversity and composition of microbial communities, but whether microbiomes from different environments exhibit similar degrees of resistance or rates of recovery has not been evaluated. Here, we synthesized 86 time series of disturbed mammalian, aquatic, and soil microbiomes to examine how the recovery of microbial richness and community composition differed after disturbance. We found no general patterns in compositional variance (i.e., dispersion) in any microbiomes over time. Only mammalian microbiomes consistently exhibited decreases in richness following disturbance. Importantly, they tended to recover this richness, but not their composition, over time. In contrast, aquatic microbiomes tended to diverge from their pre-disturbance composition following disturbance. By synthesizing microbiome responses across environments, our study aids in the reconciliation of disparate microbial community assembly frameworks, and highlights the role of the environment in microbial community reassembly following disturbance.

\section{INTRODUCTION}

Disturbances to ecological communities (i.e., externally imposed forces that cause mortality or stress to at least some of the members in the community) represent a critical process that influences the diversity and composition of taxa in those communities (Rykiel 1985). The resistance, resilience and invariance of communities following disturbances over time (Clarket al. 2021) provide insight into how ecological communities reassemble in the face of natural and anthropogenic disturbances, which are growing in frequency and magnitude (Newman 2019). The study of metacommunity assembly seeks to understand how taxa co-occur in local communities and the regions in which they are embedded, and how this varies through time, such as in the face of external perturbations (e.g., (Leibold \& Chase 2017).

There are a multitude of ways to quantify how metacommunities respond to, or recover from disturbance, each of which gives insights into different parts of the community assembly process (overviewed in Figure 1, see also (Philippotet al. 2021). First, we can measure summary variables such as taxa richness in a given locality immediately following the disturbance, which gives us a picture of the resistance of the community to disturbance. Over longer periods of time following the disturbance, measuring richness can lend insight into the recovery of the community. Across both aquatic and terrestrial ecosystems, richness is found to decline at local scales after disturbance (Murphy \& Romanuk 2014), and similar patterns have been observed in microbial communities (Shadeet al. 2012a). However, richness can also increase if competitively dominant 
taxa are more strongly depressed by the disturbance (Kondoh 2001), or in microbes, if the disturbance involves the addition of novel taxa (e.g., with sewage sludge amendments to soil (Hoet al. 2017). Over longer time scales, richness may either fail to fully recover (at least within the period observed; e.g., (Pillaet al. 2020), recover fully (Hillebrand \& Kunze 2020), or even be higher following disturbance (Hartmannet al. 2015).

Second, we can measure compositional variation among local communities following disturbance. Compositional dispersion can be taken as an indicator of beta-diversity (Andersonet al. 2006). Following disturbance, dispersion can decrease, for example, if only a subset of taxa can persist and/or priority effects are reduced (Chase 2003, 2007). Alternatively, dispersion can increase, for example, if different taxa are favored due to stochastic differences in which taxa persist following disturbances (Debrayet al. 2021). In microbial ecology, this observation of change in dispersion following disturbance has been well documented (e.g., (Zaneveldet al. 2017).

Third, we can measure to what extent community composition recovers following disturbance. The tendency of the community to return to its pre-disturbance conformation, or to tend away from this original conformation over time (i.e., distance from undisturbed controls) can be quantified viaturnover. Given enough time, we might expect the same taxa that dominated prior to a disturbance to recover to their original abundances (Philippotet al. 2021). However, under some circumstances (e.g., strong or long disturbances, invasion (Ratajczaket al. 2017; Amor et al. 2020), it is also possible that the disturbance could permanently alter dominance patterns in the community (e.g., (Seekatzet al. 2015; Khan et al. 2019). Using a null modelling approach, it is possible to further partition observed changes in dispersion and turnover into compositional and richness changes (Chaseet al. 2011) and shed light on the processes driving microbial community recovery.

Evidence for the effect of disturbance on the dispersion and turnover of microbial communities is mixed and environment-dependent. For example, the recovery of disturbed animal-associated microbiomes has been studied in relation to host health. It has been suggested that the microbiomes of sick hosts generally exhibit greater dispersion than healthy or undisturbed microbiomes (Zaneveldet al. 2017), but experimental research has found that disturbance can either reduce (Lavrinienkoet al. 2020) or increase dispersion in hostassociated microbiomes (Neely et al. 2021), and that this pattern is dependent on the time since disturbance (Ferrenberget al. 2013). Across environments, microbiomes have been shown to recover towards (negative turnover, e.g., (Shadeet al. 2012b; Jurburg et al. 2017), or to drift away from (positive turnover, e.g., (Shawet al. 2019), their pre-disturbance compositions. Among other factors, different microbial habitats have varying degrees of spatial and temporal heterogeneity, microbial species pool sizes, and resource availability, which may affect community reassembly processes and result in different patterns among environments. For example, animal gut microbiomes have relatively low diversity (Thompsonet al. 2017) and are dispersal-limited due to the host physiology, likely affecting the recovery of diversity. In contrast, soil microbiomes are extremely diverse, but poorly connected (Vos et al. 2013), likely affecting local dispersal and recolonization following disturbance.

Studying the responses of microbial communities through a metacommunity assembly lens (Leibold \& Chase 2017) can yield a generalized understanding of how microbial communities assemble following disturbance across environments, which is a pressing question in microbial ecology (Philippotet al. 2021). Unique characteristics of microbes, including rapid evolution (Niehuset al. 2015), extreme taxonomic richness (Shoemaker et al. 2017), functional redundancy (Curtis \& Sloan 2004), and dormancy (Loceyet al. 2020) may result in deviations from the patterns observed in eukaryotic systems.

A better understanding of how microbiomes assemble following disturbance could also aid in the development of treatments of diseases in plants and animals, allow us to anticipate the effect of environmental change on the soil biota, and contribute towards developing novel practices for microbiome management (Konopkaet al. 2015). To assess commonalities and differences in microbiome disturbance responses across environments, we performed a synthetic analysis of each of the responses discussed above (richness, dispersion and turnover) in the short and long term response to disturbance in aquatic, mammal-associated, and soil microbiomes. 
Given the rapid rates of compositional turnover in many microbiomes (Kenneyet al. 2020), we focused on studies that repeatedly sampled the microbiomes within 50 days post disturbance.

\section{MATERIALS AND METHODS}

\section{Dataset selection}

Using Google Scholar and Web of Science (a list of keywords is available as Supplementary Materials), we collected studies from each system where an experimental disturbance was imposed and 16S rRNA gene amplicon sequencing datasets were available. Specifically, we chose studies, which : 1) were sequenced in Illumina or IonTorrent platforms; 2) sequenced the v3-v4 regions of the 16S rRNA gene; 3) were published after 2014 ; 4) repeatedly sampled microbial communities following a discrete disturbance or environmental change; 5) included samples from before the disturbance (i.e., controls), at least one sampling within a week after disturbance, and at least one sampling within a month after disturbance; and 6) included experimental triplicates (i.e., three samples per time point). Criteria 1-3 ensured that the sequencing techniques were comparable between studies, and avoided the biases associated with sampling different regions of the 16S rRNA gene (Yanget al. 2016). Whether shifts in community composition occur at similar rates across environments is seldom compared using empirical data. Criteria 4-6 ensured that the time scales were comparable between time series, that the effects of environmental change could be comparedwithin time series between different time points, and that the variability of the microbiomes at each time point could be measured. We defined a pulse disturbance as a "discrete, short-term event" (Shadeet al. 2012a). We excluded datasets for which raw sequencing data was not publicly available, and stopped data collection in (Hoet al. 2017) October 2020. In all, 29 datasets matched our criteria (Davidet al. 2015; Seekatz et al. 2015; Datta et al.2016; Džunková et al. 2016; Fuentes et al. 2016; Vaquer-Sunyer et al. 2016; Venkataraman et al. 2016; Wuet al. 2016; Dong et al. 2017; Jurburg et al. 2017, 2018, 2019; Qian et al. 2017; de Vries et al. 2018; Flancman et al. 2018; Frenk et al. 2018; Kennedy et al. 2018; Li et al. 2018, 2019; Mateos et al. 2018; van Kruistum et al. 2018; Lavelle et al. 2019; Lu et al. 2019; Santi et al. 2019; Ward et al. 2019; Yanet al. 2020) Table S1). We grouped these time series into three environmental categories: aquatic, mammal-associated, and soil microbiomes (including rhizosphere microbiomes).

\section{Sequence reprocessing}

We obtained raw 16S rRNA gene amplicon data and metadata from the NCBI Sequence Read Archive with the exception of two datasets, one of which came from another database, and the other was obtained directly from the authors (see Table S1 for accession numbers). We processed sequences in R 3.4.3 (Team 2017) using the dada2 package (Callahanet al. 2016a). Prior to processing, we visually inspected two sequences per study with the plotQualityProfile to determine whether the reads had been merged prior to archiving and to confirm that primers were not present. We only used forward reads because reverse reads were not available for all studies. Following inspection, we trimmed and truncated sequences on a study-by-study basis (see Table S1 for trimming and truncation lengths) to preserve a $90 \mathrm{bp}$ segment, the minimum recommended in the Earth Microbiome Project protocols (Thompsonet al. 2017). We filtered, dereplicated, and chimerachecked each read using standard workflow parameters (Callahanet al. 2016b), and assigned reads to exact sequence variants (ASVs, 100\% sequence identity in the $90 \mathrm{bp}$ segment) with the SILVA v.132 training set (Quastet al. 2013). We removed reads not assigned to the domain Bacteria. Details about the percentage of reads lost at each step of sequence processing, per study, are included in Figure S1. Due to the wide range of sequencing depths across samples, we standardized samples to 1,500 reads per sample. To ensure that our findings were not affected by observation depth, we ran all analyses in parallel using the deepest possible observation depth (with a lower bound of 1,500 reads per sample) for each study (Table S1). As our findings were consistent regardless of standardization (Figure S2), and we present only the results from the global standardization. To examine the completeness of each sample relative to the total richness in a community, we calculated coverage (Chao and Jost 2012) using the BetaC package (Engelet al. 2020). On average, our samples represented $0.96+-0.05$ (mean + -sd) of the community. We removed any time points that had less than three experimental replicates for each time series. We coded time series so that time (days) [?] 0 occurred after disturbance, and time $<0$ denoted the pre-disturbance community. In line 
with our conservative approach to sequence processing, our analyses focused on the dominant portion of the community.

\section{Calculation of richness and turnover metrics}

We calculated richness and turnover metrics using the phyloseqpackage's data structure (McMurdie \& Holmes 2013). We calculated species richness as the number of unique ASVs per sample.

We used Bray-Curtis distances to quantify two aspects of compositional variation. First, for each time series and for each time point, we calculated dispersion as the pairwise Bray-Curtis distances between all combinations of experimental replicates. For studies that resampled the same experimental unit (e.g., host organism or microcosm) over time, we excluded pairwise comparisons between samples from the same experimental units. Second, to quantify turnover, we calculated pairwise distances between all control samples (i.e., pre-disturbance) and all subsequent replicate samples at each time point following disturbance. Communities that largely recover to their pre-disturbance conformation will have a negative slope through time, while communities that become increasingly different from the pre-disturbance community over time will have a positive slope estimate (Figure 1).

To disentangle compositional changes from changes in richness, we randomly permuted abundance values within each sample 1000 times, preserving the number of taxa and observations for each sample, and recalculated turnover and dispersion metrics for each matrix to derive a null expectation for each. For both metrics, Z-scores were calculated as $\frac{\text { observed-rexpected }}{\sigma^{\text {expected }}}$. We present analyses of the Z-scores in the main text, and analyses of the raw compositional metrics (i.e., Bray-Curtis) in the Supplementary Methods. All code for bioinformatics processing and null models is available at https://github.com/drcarrot/DisturbanceSynthesis.

\section{Statistical analyses}

We fit statistical models to assess how 1) richness, 2) dispersion, and 3) turnover change in response to the disturbance relative to undisturbed control samples immediately after disturbance (i.e., before-after analyses), and through time. Unless otherwise specified, we performed all analyses at the ASV level. Beforeafter analyses compared data from prior to the disturbance to samples taken $<4$ days post disturbance, and models included before-after and environment (i.e, aquatic, mammal, soil) as categorical covariates that were allowed to interact. A list of the studies excluded from before-after analyses due to lack of samples is included in Table S1. We assessed diversity change through time with models fit to data from the first 50 days post-disturbance only (i.e., pre-disturbance samples were not included, with the exception of the turnover analysis that quantified the compositional change between pre-disturbance and post-disturbance assemblages). We fit time series models with time (in days) as a continuous covariate; time was centered by subtracting the mean duration from all observations prior to modelling and allowed to interact with the environment. We fit all models with the same, hierarchical grouping (or random-effects) structure. To account for methodological variation between studies, we included varying intercepts in all models; and, because many studies included more than one disturbance type (e.g., (Džunkováet al. 2016), we included varying slopes and intercepts for time series within studies (i.e., one time series per disturbance type).

We assumed a negative-binomial error distribution and a log-link function for models fit to species richness calculations (i.e., the before-after and time series models). In addition to the parameters and the grouping structure described above, the shape parameter of the negative-binomial distribution (that estimates aggregation) was also allowed to vary among studies. Models fit to the z-transformed compositional change metrics (i.e., Bray-Curtis) assumed Gaussian error distributions and identity-link functions. In addition to the parameters and grouping structure described above, we also modelled residual variation as a function of environment to account for heteroscedasticity, with additional random variation among studies also modelled.

For Bayesian inference and estimates of uncertainty, we fit models using the Hamiltonian Monte Carlo (HMC) sampler Stan (Carpenter et al. 2017), which were coded using the 'brms' package (Bürkner 2017). We used weakly regularising priors, and visual inspection of the HMC chains showed excellent convergence. All code 
for statistical analyses is available at https://github.com/sablowes/microbiome-disturbance.

\section{RESULTS}

Our dataset included 2588 samples in 86 time series from 29 studies (Table S1) belonging to soil micro- and mesocosms $(n=49)$, seawater mesocosms $(n=16)$, and mammalian microbiomes $(n=21)$ that were sampled multiple times within 50 days after disturbance (Figure 2a). Across all samples, we detected 56,480 ASVs. Of the 824 families in our dataset, $85.6 \%$ were detected in seawater, mammalian, and soil microbiomes (Figure S3). Coverage was highest in mammalian microbiomes $(0.98 \pm 0.02$; mean \pm sd), lowest and most variable in soil microbiomes $(0.93 \pm 0.06)$, and was significantly different between environments (ANOVA, $F=475.1$, $\mathrm{p}<0.001$, Figure 2b).

\section{Richness in disturbed and recovering microbiomes}

Prior to disturbance, mean richness was highest in soil microbiomes with 327 ASVs [95\% CI: 196 - 506] , followed by aquatic 184 [111- 281], and mammalian 86 [51 - 133] microbiomes (Figure 3a). While all environments exhibited slight decreases in microbiome richness following disturbance, only the decrease in the mammalian microbiomes differed from zero. On average, the post-disturbance richness in mammalian microbiomes was approximately $43 \%$ of that found pre-disturbance (Figure 3a), and over time, richness increased approximately $2 \%(1-3 \%)$ per day (Figure $3 \mathrm{~b}$ ). In general, the mammalian microbiomes that lost the most richness after disturbance also recovered this richness most rapidly over the following 50 days (Figure S4).

\section{Dispersion and turnover}

All microbial communities were underdispersed relative to the null expectation, and $97 \%$ of Z-scores were negative. On average, dispersion did not change in response to disturbance for any environment, either immediately or over time (Figure 4, Table S2). We found a decrease in the Bray-Curtis dispersion values for mammalian microbiomes (Figure S5), however this pattern was not present in the Z-scores, indicating that this decrease was due to losses in richness rather than compositional change.

Following disturbance, aquatic microbiomes exhibited positive turnover, tending away from their predisturbance controls over time (slope estimate: 0.06 [0.04-0.08]). No temporal patterns were observed in mammalian (slope estimate: -0.02 [-0.04- -0.01]; Figure 5,6) or soil (slope estimate: 0 [-0.01- 0.02]) microbiomes. We found that mammalian microbiomes exhibited negative turnover when assessed with the raw Bray-Curtis values, tending to recover to their pre-disturbance composition. However, once again, our null model confirmed that this pattern was due to an increase in richness, not due to compositional recovery (Figure S6). Immediate richness loss was not directly related to turnover responses over time, however both parameters were environment-dependent (Figure 6).

\section{DISCUSSION}

By investigating patterns of microbial community reassembly at time scales that are relevant to microbiome turnover rates and bacterial life histories (Shadeet al. 2018; Kenney et al. 2020), our study takes advantage of relatively standardized and available microbiome data (i.e., the V3-V4 region of the $16 \mathrm{~S}$ rRNA marker gene, (Jurburget al. 2020). We found that in general, microbiomes experienced modest richness losses and no change in dispersion following disturbance. We also found environment-specific responses: aquatic microbiomes tended away from their pre-disturbance composition following disturbance, while mammalian and soil microbiomes exhibited no clear patterns. These findings can help reconcile disparate observations previously noted in the literature.

Contrary to our expectation, we only found modest losses in richness following disturbance. On average, only mammalian microbiomes experienced significant richness loss. This loss likely underscores the efficacy of antibiotics and other targeted treatments, which were the disturbance most frequently used to induce disturbances in mammalian microbiomes (Seekatzet al. 2015; Džunková et al. 2016); disturbances in the other environments in our study tended to be untargeted (e.g., inorganic nitrogen and phosphorus inputs 
in aquatic microbiomes, (Santiet al. 2019); or humic acid amendments in soil, (Liet al. 2019). The host's response may have also played a role in both the immediate response and the long-term recovery of richness following disturbance. Host responses depend on the degree of richness loss, the identity of the lost taxa (Yaoet al. 2016), and the intensity of disturbance (Van de Guchte et al. 2020), and can exacerbate the effect of disturbance on the microbiome (e.g., through inflammation (Van de Guchte et al. 2020). We found that more strongly disturbed mammalian microbiomes tended to recover their richness more rapidly, and here, too, host behaviors such as eating (Guptaet al. 2017) and socializing (Rauloet al. 2021) may have functioned as mechanisms of active dispersal. The mild decline in richness following disturbance in aquatic and soil microbiomes contrasts the stronger responses found in eukaryotic communities to disturbance (Murphy \& Romanuk 2012, 2014). This may be because the disturbances applied in these communities only mildly affected them, or because rare taxa were disproportionately affected and we were unable to detect their decrease due to the conservative approaches selected for sequence processing.

Our work further highlights the need to reframe the consequences of disturbances in the microbiome (Buma 2021). We included a wide range of disturbances that are not usually considered in the eukaryotic literature. For example, when sterilized, organic amendments represent a novel source of resources, but when applied unsterilized, they also include a potentially invasive community, a scenario that deviates from the classic invasion literature (Rilliget al. 2015). Furthermore, the duration of disturbances varied, especially relative to bacterial life histories and ecologies (Kenneyet al. 2020). Pulse disturbances which last multiple days may encompass multiple life cycles for many microbial taxa. Similarly, disturbances which may be considered long-term changes for macroorganisms (i.e., oil pollution), may represent short-term resource pulses for oil-degrading bacteria. In a world in which microbiomes are exposed to increasing disturbance pressures, developing a set of descriptors for disturbances based on their effect on the microbiome's niche space and competitive landscape is urgently needed.

We found no patterns in dispersion, either immediately after or over time following disturbance, in any environment. While changes in dispersion are often reported in the microbial literature (Ferrenberget al. 2013; Lavrinienko et al. 2020; Neely et al.2021), dispersion is generally measured as pairwise Bray-Curtis distances among experimental or field replicates, and thus confound changes in richness with compositional changes (Chaseet al. 2011; Stegen et al. 2013). Our null modelling approach allowed us to partition these two sources of change, revealing that in general. compositional dispersion does not consistently increase or decrease following disturbance, even when richness changes (i.e., in mammalian microbiomes). In particular, despite recovering their richness, mammalian microbiomes did not recover their composition, in line with findings across a wide range of ecosystems (Hillebrand \& Kunze 2020). Whether these altered microbial community compositions are important for the maintenance of community functions (Nemergutet al. 2014) and resilience (Paineet al. 1998) is an open, but pressing issue.

Surprisingly, aquatic microbiomes tended to become more dissimilar from their pre-disturbance compositions over time. Due to the different temporal sampling schemes included in this synthesis, it is not possible to determine whether the communities were generally drifting towards a specific composition. The observed pattern may be due to the high connectivity of aquatic microbiomes (Mestreet al. 2018), where dispersal limitation is greatly reduced relative to other microbiomes and nutrients are constantly mixed, facilitating the random recolonization of taxa following disturbance. Indeed, in the highly heterogeneous soil environment, microbiomes exhibited little consistent responses to disturbance, likely due to the extreme diversity and heterogeneity found in this system (Rilliget al. 2017), or due to technical limitations of this study. The conservative approaches we employed for the selection, processing and analysis of the data aimed to facilitate cross-study comparisons, but limited the contribution of rare or lowly abundant taxa to our analysis. This effect was likely stronger for soil, which has the highest overall richness (Thompsonet al. 2017), where our sample completeness estimates (i.e., coverage) were lowest. Rare taxa are important sources of variation in soil microbiomes (Joussetet al. 2017; Jiao et al. 2019), and are key drivers of community assembly (Shadeet al. 2014).

Our synthesis is intended to explicitly compare microbial community reassembly patterns in response to 
disturbance across realms that are highly segmented by researcher identity. Our study reconciles several hypotheses that have been proposed for microbiomes in the different realms. First, we find support for the tendency to drift away from the pre-disturbance condition in aquatic systems. Second, we find that mammalian microbiomes often recover their richness, but not their composition following disturbance (e.g., they maintain the same number of taxa but have different memberships with different relative contributions). Third, we find little general evidence for changes in compositional dispersion (after accounting for changes in richness) following disturbance. Finally, our study reframes microbial community reassembly from a community ecology perspective. Future work should focus on distinguishing targeted from untargeted disturbances, to determine what constitutes a disturbance for a microbiome given its ecosystem context, and to perform research at temporal scales which match microbial life histories and turnover /growth in microbiomes. Overall, this work provides a new perspective on the dynamics and generalities of microbiome disturbance responses that is supported by directly comparable metrics, equivalent temporal scales among datasets, and a consistent modeling approach. It suggests that, after standardizing and focusing on macroecological patterns, the environment matters for microbiome assembly.

\section{ACKNOWLEDGEMENTS}

We would like to thank A. Heintz-Buschart for their help and S. Tem and A Clark for valuable discussions. We acknowledge support by the German Centre for Integrative Biodiversity Research Halle-Jena-Leipzig, funded by the German Research Foundation (FZT 118, 202548816) and the synthesis centre of iDiv (sDiv). The study has in part been performed using the High-Performance Computing (HPC) Cluster EVE, a joint effort of both the Helmholtz Centre for Environmental Research - UFZ and the German Centre for Integrative Biodiversity Research (iDiv) Halle-Jena-Leipzig. AS acknowledges support from National Science Foundation CAREER (NSF 1749544).

Bibliography

Amor, D.R., Ratzke, C. \& Gore, J. (2020). Transient invaders can induce shifts between alternative stable states of microbial communities. Sci. Adv., 6, eaay8676.

Anderson, M.J., Ellingsen, K.E. \& McArdle, B.H. (2006). Multivariate dispersion as a measure of beta diversity. Ecol. Lett., 9, 683-693.

Buma, B. (2021). Disturbance ecology and the problem of $n=1$ : A proposed framework for unifying disturbance ecology studies to address theory across multiple ecological systems. Methods Ecol. Evol.

Burkner, P.-C. (2017). brms: an $R$ package for bayesian multilevel models using stan. J. Stat. Softw., 80.

Callahan, B.J., McMurdie, P.J., Rosen, M.J., Han, A.W., Johnson, A.J.A. \& Holmes, S.P. (2016a). DADA2: High-resolution sample inference from Illumina amplicon data. Nat. Methods, 13, 581-583.

Callahan, B.J., Sankaran, K., Fukuyama, J.A., McMurdie, P.J. \& Holmes, S.P. (2016b). Bioconductor Workflow for Microbiome Data Analysis: from raw reads to community analyses. [version 2; peer review: 3 approved].F1000Res., 5, 1492.

Carpenter, B., Gelman, A., Hoffman, M.D., Lee, D., Goodrich, B., Betancourt, M.,et al. (2017). Stan: a probabilistic programming language. Grantee Submission, 76, 1-32.

Chase, J.M., Kraft, N.J.B., Smith, K.G., Vellend, M. \& Inouye, B.D. (2011). Using null models to disentangle variation in community dissimilarity from variation in $\alpha$-diversity. Ecosphere, 2, art24.

Chase, J.M. (2003). Community assembly: when should history matter? Oecologia, 136, 489-498.

Chase, J.M. (2007). Drought mediates the importance of stochastic community assembly. Proc Natl Acad Sci USA, 104, 17430-17434.

Clark, A.T., Arnoldi, J.-F., Zelnik, Y.R., Barabas, G., Hodapp, D., Karakoç, C.,et al. (2021). General statistical scaling laws for stability in ecological systems. Ecol. Lett., 24, 1474-1486. 
Curtis, T.P. \& Sloan, W.T. (2004). Prokaryotic diversity and its limits: microbial community structure in nature and implications for microbial ecology.Curr. Opin. Microbiol., 7, 221-226.

Datta, M.S., Sliwerska, E., Gore, J., Polz, M.F. \& Cordero, O.X. (2016). Microbial interactions lead to rapid micro-scale successions on model marine particles. Nat. Commun., 7, 11965.

David, L.A., Weil, A., Ryan, E.T., Calderwood, S.B., Harris, J.B., Chowdhury, F., et al. (2015). Gut microbial succession follows acute secretory diarrhea in humans. MBio, 6, e00381-15.

Debray, R., Herbert, R.A., Jaffe, A.L., Crits-Christoph, A., Power, M.E. \& Koskella, B. (2021). Priority effects in microbiome assembly. Nat. Rev. Microbiol.

Dong, Z., Wang, K., Chen, X., Zhu, J., Hu, C. \& Zhang, D. (2017). Temporal dynamics of bacterioplankton communities in response to excessive nitrate loading in oligotrophic coastal water. Mar. Pollut. Bull., 114, 656-663.

Džunková, M., D’Auria, G., Xu, H., Huang, J., Duan, Y., Moya, A., et al.(2016). The Monoclonal Antitoxin Antibodies (Actoxumab-Bezlotoxumab) Treatment Facilitates Normalization of the Gut Microbiota of Mice with Clostridium difficile Infection. Front. Cell. Infect. Microbiol., 6, 119.

Engel, T., Blowes, S.A., McGlinn, D.J., May, F., Gotelli, N.J., McGill, B.J., et al. (2020). Using coverage-based rarefaction to infer non-random species distributions. BioRxiv.

Ferrenberg, S., O’Neill, S.P., Knelman, J.E., Todd, B., Duggan, S., Bradley, D.,et al. (2013). Changes in assembly processes in soil bacterial communities following a wildfire disturbance. ISME J., 7, 1102-1111.

Flancman, R., Singh, A. \& Weese, J.S. (2018). Evaluation of the impact of dental prophylaxis on the oral microbiota of dogs. PLoS ONE, 13, e0199676.

Frenk, S., Hadar, Y. \& Minz, D. (2018). Quality of irrigation water affects soil functionality and bacterial community stability in response to heat disturbance. Appl. Environ. Microbiol., 84.

Fuentes, S., Barra, B., Caporaso, J.G. \& Seeger, M. (2016). From Rare to Dominant: a Fine-Tuned Soil Bacterial Bloom during Petroleum Hydrocarbon Bioremediation. Appl. Environ. Microbiol., 82, 888-896.

Gupta, V.K., Paul, S. \& Dutta, C. (2017). Geography, Ethnicity or Subsistence-Specific Variations in Human Microbiome Composition and Diversity. Front. Microbiol., 8, 1162.

Hartmann, M., Frey, B., Mayer, J., Mäder, P. \& Widmer, F. (2015). Distinct soil microbial diversity under long-term organic and conventional farming.ISME J., 9, 1177-1194.

Hillebrand, H. \& Kunze, C. (2020). Meta-analysis on pulse disturbances reveals differences in functional and compositional recovery across ecosystems.Ecol. Lett., 23, 575-585.

Ho, A., Ijaz, U.Z., Janssens, T.K.S., Ruijs, R., Kim, S.Y., de Boer, W., et al.(2017). Effects of bio-based residue amendments on greenhouse gas emission from agricultural soil are stronger than effects of soil type with different microbial community composition. Glob. Change Biol. Bioenergy.

Jiao, S., Wang, J., Wei, G., Chen, W. \& Lu, Y. (2019). Dominant role of abundant rather than rare bacterial taxa in maintaining agro-soil microbiomes under environmental disturbances. Chemosphere, 235, 248-259.

Jousset, A., Bienhold, C., Chatzinotas, A., Gallien, L., Gobet, A., Kurm, V., et al. (2017). Where less may be more: how the rare biosphere pulls ecosystems strings. ISME J., 11, 853-862.

Jurburg, S.D., Cornelissen, J.J.B.W.J., de Boer, P., Smits, M.A. \& Rebel, J.M.J. (2019). Successional Dynamics in the Gut Microbiome Determine the Success of Clostridium difficile Infection in Adult Pig Models.Front. Cell. Infect. Microbiol., 9, 271.

Jurburg, S.D., Konzack, M., Eisenhauer, N. \& Heintz-Buschart, A. (2020). The archives are half-empty: an assessment of the availability of microbial community sequencing data. Commun. Biol., 3, 474. 
Jurburg, S.D., Natal-da-Luz, T., Raimundo, J., Morais, P.V., Sousa, J.P., van Elsas, J.D., et al. (2018). Bacterial communities in soil become sensitive to drought under intensive grazing. Sci. Total Environ., 618, 1638-1646.

Jurburg, S.D., Nunes, I., Stegen, J.C., Le Roux, X., Priemé, A., Sørensen, S.J.,et al. (2017). Autogenic succession and deterministic recovery following disturbance in soil bacterial communities. Sci. Rep., 7, 45691.

Kennedy, R.C., Fling, R.R., Robeson, M.S., Saxton, A.M., Schneider, L.G., Darcy, J.L., et al. (2018). Temporal dynamics of gut microbiota in triclocarban-exposed weaned rats. Environ. Sci. Pollut. Res. Int., 25, 14743-14751.

Kenney, T., Gao, J. \& Gu, H. (2020). Application of OU processes to modelling temporal dynamics of the human microbiome, and calculating optimal sampling schemes. BMC Bioinformatics, 21, 450.

Khan, M.J., Jurburg, S.D., He, J., Brodie, G. \& Gupta, D. (2019). Impact of microwave disinfestation treatments on the bacterial communities of no-till agricultural soils. European Journal of Soil Science.

Kondoh, M. (2001). Unifying the relationships of species richness to productivity and disturbance. Proc. Biol. Sci., 268, 269-271.

Konopka, A., Lindemann, S. \& Fredrickson, J. (2015). Dynamics in microbial communities: unraveling mechanisms to identify principles. ISME J., 9, 1488-1495.

van Kruistum, H., Bodelier, P.L.E., Ho, A., Meima-Franke, M. \& Veraart, A.J. (2018). Resistance and Recovery of Methane-Oxidizing Communities Depends on Stress Regime and History; A Microcosm Study. Front. Microbiol., 9, 1714.

Lavelle, A., Hoffmann, T.W., Pham, H.-P., Langella, P., Guedon, E. \& Sokol, H. (2019). Baseline microbiota composition modulates antibiotic-mediated effects on the gut microbiota and host. Microbiome, 7, 111.

Lavrinienko, A., Tukalenko, E., Kesaniemi, J., Kivisaari, K., Masiuk, S., Boratyński, Z.,et al. (2020). Applying the Anna Karenina principle for wild animal gut microbiota: Temporal stability of the bank vole gut microbiota in a disturbed environment. J. Anim. Ecol., 89, 2617-2630.

Leibold, M.A. \& Chase, J.M. (2017). Metacommunity Ecology, Volume 59 (Monographs in Population Biology, 59). Princeton University Press, Princeton, NJ.

Li, L., Wang, S., Li, X., Li, T., He, X. \& Tao, Y. (2018). Effects of Pseudomonas chenduensis and biochar on cadmium availability and microbial community in the paddy soil. Sci. Total Environ., 640-641, 1034-1043.

Li, P., Liu, J., Jiang, C., Wu, M., Liu, M. \& Li, Z. (2019). Distinct Successions of Common and Rare Bacteria in Soil Under Humic Acid Amendment - A Microcosm Study. Front. Microbiol., 10, 2271.

Locey, K.J., Muscarella, M.E., Larsen, M.L., Bray, S.R., Jones, S.E. \& Lennon, J.T. (2020). Dormancy dampens the microbial distance-decay relationship.Philos. Trans. R. Soc. Lond. B Biol. Sci., 375, 20190243.

Lu, T., Zhou, Z., Zhang, Q., Zhang, Z. \& Qian, H. (2019). Ecotoxicological effects of fungicides azoxystrobin and pyraclostrobin on freshwater aquatic bacterial communities. Bull. Environ. Contam. Toxicol., 103, 683-688.

Mateos, I., Combes, S., Pascal, G., Cauquil, L., Barilly, C., Cossalter, A.-M.,et al. (2018). FumonisinExposure Impairs Age-Related Ecological Succession of Bacterial Species in Weaned Pig Gut Microbiota.Toxins (Basel), 10.

McMurdie, P.J. \& Holmes, S. (2013). phyloseq: an R package for reproducible interactive analysis and graphics of microbiome census data. PLoS ONE, 8, e61217.

Mestre, M., Ruiz-González, C., Logares, R., Duarte, C.M., Gasol, J.M. \& Sala, M.M. (2018). Sinking particles promote vertical connectivity in the ocean microbiome. Proc Natl Acad Sci USA, 115, E6799-E6807. 
Murphy, G.E.P. \& Romanuk, T.N. (2012). A meta-analysis of community response predictability to anthropogenic disturbances. Am. Nat., 180, 316-327.

Murphy, G.E.P. \& Romanuk, T.N. (2014). A meta-analysis of declines in local species richness from human disturbances. Ecol. Evol., 4, 91-103.

Neely, W.J., Greenspan, S.E., Stahl, L.M., Heraghty, S.D., Marshall, V.M., Atkinson, C.L., et al. (2021). Habitat Disturbance Linked with Host Microbiome Dispersion and Bd Dynamics in Temperate Amphibians. Microb. Ecol.

Nemergut, D.R., Shade, A. \& Violle, C. (2014). When, where and how does microbial community composition matter? Front. Microbiol., 5, 497.

Newman, E.A. (2019). Disturbance ecology in the anthropocene. Front. Ecol. Evol., 7.

Niehus, R., Mitri, S., Fletcher, A.G. \& Foster, K.R. (2015). Migration and horizontal gene transfer divide microbial genomes into multiple niches. Nat. Commun., 6, 8924.

Paine, R.T., Tegner, M.J. \& Johnson, E.A. (1998). Compounded Perturbations Yield Ecological Surprises. Ecosystems, 1, 535-545.

Philippot, L., Griffiths, B.S. \& Langenheder, S. (2021). Microbial Community Resilience across Ecosystems and Multiple Disturbances. Microbiol. Mol. Biol. Rev., 85.

Pilla, R., Gaschen, F.P., Barr, J.W., Olson, E., Honneffer, J., Guard, B.C., et al. (2020). Effects of metronidazole on the fecal microbiome and metabolome in healthy dogs. J. Vet. Intern. Med., 34, 1853-1866.

Qian, J., Ding, Q., Guo, A., Zhang, D. \& Wang, K. (2017). Alteration in successional trajectories of bacterioplankton communities in response to co-exposure of cadmium and phenanthrene in coastal water microcosms. Environ. Pollut., 221, 480-490.

Quast, C., Pruesse, E., Yilmaz, P., Gerken, J., Schweer, T., Yarza, P., et al.(2013). The SILVA ribosomal RNA gene database project: improved data processing and web-based tools. Nucleic Acids Res., 41, D590-6.

Ratajczak, Z., D’Odorico, P., Collins, S.L., Bestelmeyer, B.T., Isbell, F.I. \& Nippert, J.B. (2017). The interactive effects of press/pulse intensity and duration on regime shifts at multiple scales. Ecol. Monogr., $87,198-218$.

Raulo, A., Allen, B.E., Troitsky, T., Husby, A., Firth, J.A., Coulson, T., et al.(2021). Social networks strongly predict the gut microbiota of wild mice. ISME $J$.

Rillig, M.C., Antonovics, J., Caruso, T., Lehmann, A., Powell, J.R., Veresoglou, S.D., et al. (2015). Interchange of entire communities: microbial community coalescence. Trends Ecol. Evol., 30, 470-476.

Rillig, M.C., Muller, L.A. \& Lehmann, A. (2017). Soil aggregates as massively concurrent evolutionary incubators. ISME J., 11, 1943-1948.

Rykiel, E.J. (1985). Towards a definition of ecological disturbance. Australian Journal of Ecology, 10, 361365 .

Santi, I., Tsiola, A., Dimitriou, P.D., Fodelianakis, S., Kasapidis, P., Papageorgiou, N., et al. (2019). Prokaryotic and eukaryotic microbial community responses to $\mathrm{N}$ and $\mathrm{P}$ nutrient addition in oligotrophic Mediterranean coastal waters: Novel insights from DNA metabarcoding and network analysis. Mar. Environ. Res., $150,104752$.

Seekatz, A.M., Theriot, C.M., Molloy, C.T., Wozniak, K.L., Bergin, I.L. \& Young, V.B. (2015). Fecal Microbiota Transplantation Eliminates Clostridium difficile in a Murine Model of Relapsing Disease. Infect. Immun., 83, 3838-3846. 
Shade, A., Dunn, R.R., Blowes, S.A., Keil, P., Bohannan, B.J.M., Herrmann, M., et al. (2018). Macroecology to unite all life, large and small.Trends Ecol. Evol., 33, 731-744.

Shade, A., Jones, S.E., Caporaso, J.G., Handelsman, J., Knight, R., Fierer, N., et al. (2014). Conditionally rare taxa disproportionately contribute to temporal changes in microbial diversity. MBio, 5, e01371-14.

Shade, A., Peter, H., Allison, S.D., Baho, D.L., Berga, M., Bürgmann, H., et al.(2012a). Fundamentals of microbial community resistance and resilience.Front. Microbiol., 3, 417.

Shade, A., Read, J.S., Youngblut, N.D., Fierer, N., Knight, R., Kratz, T.K., et al. (2012b). Lake microbial communities are resilient after a whole-ecosystem disturbance. ISME J., 6, 2153-2167.

Shaw, L.P., Bassam, H., Barnes, C.P., Walker, A.S., Klein, N. \& Balloux, F. (2019). Modelling microbiome recovery after antibiotics using a stability landscape framework. ISME J., 13, 1845-1856.

Shoemaker, W.R., Locey, K.J. \& Lennon, J.T. (2017). A macroecological theory of microbial biodiversity. Nat. Ecol. Evol., 1, 107.

Stegen, J.C., Lin, X., Fredrickson, J.K., Chen, X., Kennedy, D.W., Murray, C.J., et al. (2013). Quantifying community assembly processes and identifying features that impose them. ISME J., 7, 2069-2079.

Team, R.C. (2017). R: A language and environment for statistical computing. R Foundation for Statistical Computing, Vienna, Austria. 2016.

Thompson, L.R., Sanders, J.G., McDonald, D., Amir, A., Ladau, J., Locey, K.J., et al. (2017). A communal catalogue reveals Earth's multiscale microbial diversity. Nature, 551, 457-463.

Van de Guchte, M., Burz, S.D., Cadiou, J., Wu, J., Mondot, S., Blottière, H.M., et al. (2020). Alternative stable states in the intestinal ecosystem: proof of concept in a rat model and a perspective of therapeutic implications. Microbiome, 8, 153.

Vaquer-Sunyer, R., Reader, H.E., Muthusamy, S., Lindh, M.V., Pinhassi, J., Conley, D.J., et al. (2016). Effects of wastewater treatment plant effluent inputs on planktonic metabolic rates and microbial community composition in the Baltic Sea. Biogeosciences, 13, 4751-4765.

Venkataraman, A., Sieber, J.R., Schmidt, A.W., Waldron, C., Theis, K.R. \& Schmidt, T.M. (2016). Variable responses of human microbiomes to dietary supplementation with resistant starch. Microbiome, 4, 33.

de Vries, F.T., Griffiths, R.I., Bailey, M., Craig, H., Girlanda, M., Gweon, H.S., et al. (2018). Soil bacterial networks are less stable under drought than fungal networks. Nat. Commun., 9, 3033.

Ward, C.S., Pan, J.-F., Colman, B.P., Wang, Z., Gwin, C.A., Williams, T.C., et al.(2019). Conserved microbial toxicity responses for acute and chronic silver nanoparticle treatments in wetland mesocosms. Environ. Sci. Technol., 53, 3268-3276.

Wu, B., Wang, X., Yang, L., Yang, H., Zeng, H., Qiu, Y., et al. (2016). Effects of Bacillus amyloliquefaciens ZM9 on bacterial wilt and rhizosphere microbial communities of tobacco. Applied Soil Ecology, 103, 1-12.

Yang, B., Wang, Y. \& Qian, P.Y. (2016). Sensitivity and correlation of hypervariable regions in 16S rRNA genes in phylogenetic analysis. BMC Bioinformatics, 17, 135.

Yan, L., Hui, N., Simpanen, S., Tudeer, L. \& Romantschuk, M. (2020). Simulation of microbial response to accidental diesel spills in basins containing brackish sea water and sediment. Front. Microbiol., 11, 593232.

Yao, J., Carter, R.A., Vuagniaux, G., Barbier, M., Rosch, J.W. \& Rock, C.O. (2016). A Pathogen-Selective Antibiotic Minimizes Disturbance to the Microbiome.Antimicrob. Agents Chemother., 60, 4264-4273.

Zaneveld, J.R., McMinds, R. \& Vega Thurber, R. (2017). Stress and stability: applying the Anna Karenina principle to animal microbiomes. Nat. Microbiol., 2, 17121.

FIGURES 


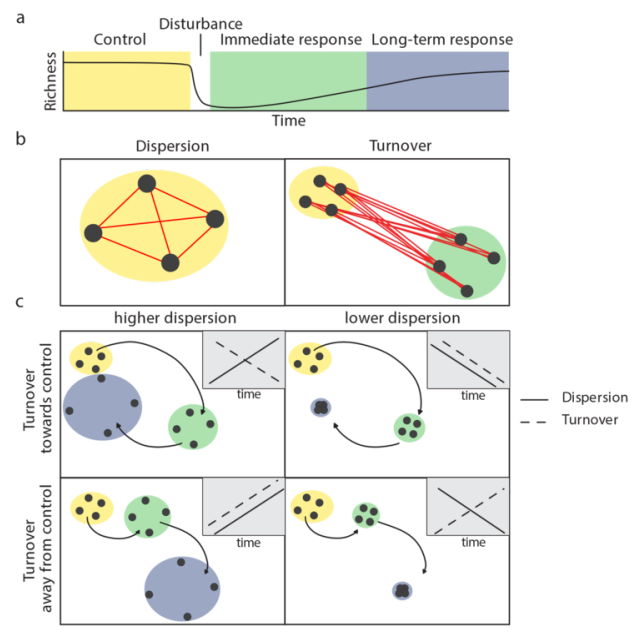

Figure 1. Measuring microbial recovery after disturbance.
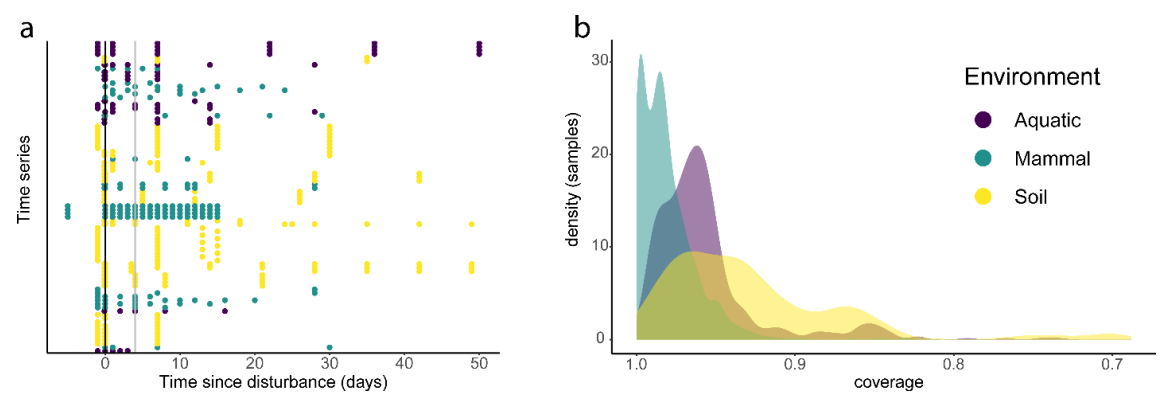

Figure 2. Samples used in this synthesis. 
a
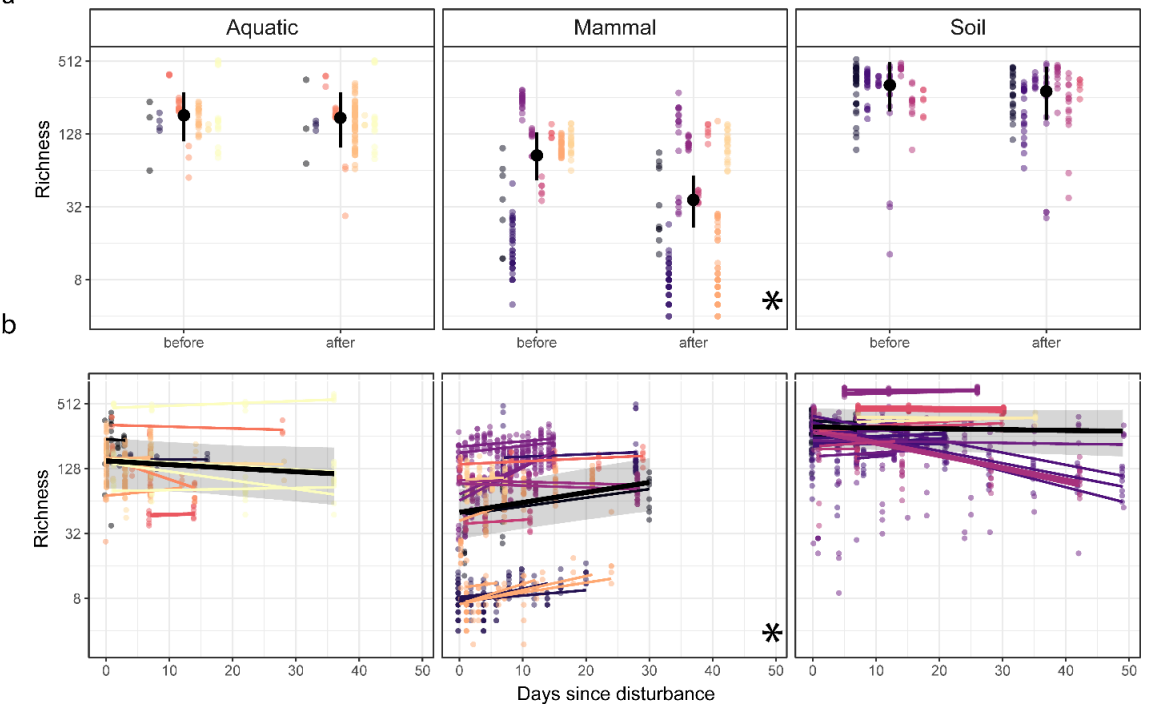

Figure 3. The effect of disturbance on microbiome richness, immediately $(<4$ days $)$ after disturbance (a), and over 50 days of recovery (b).
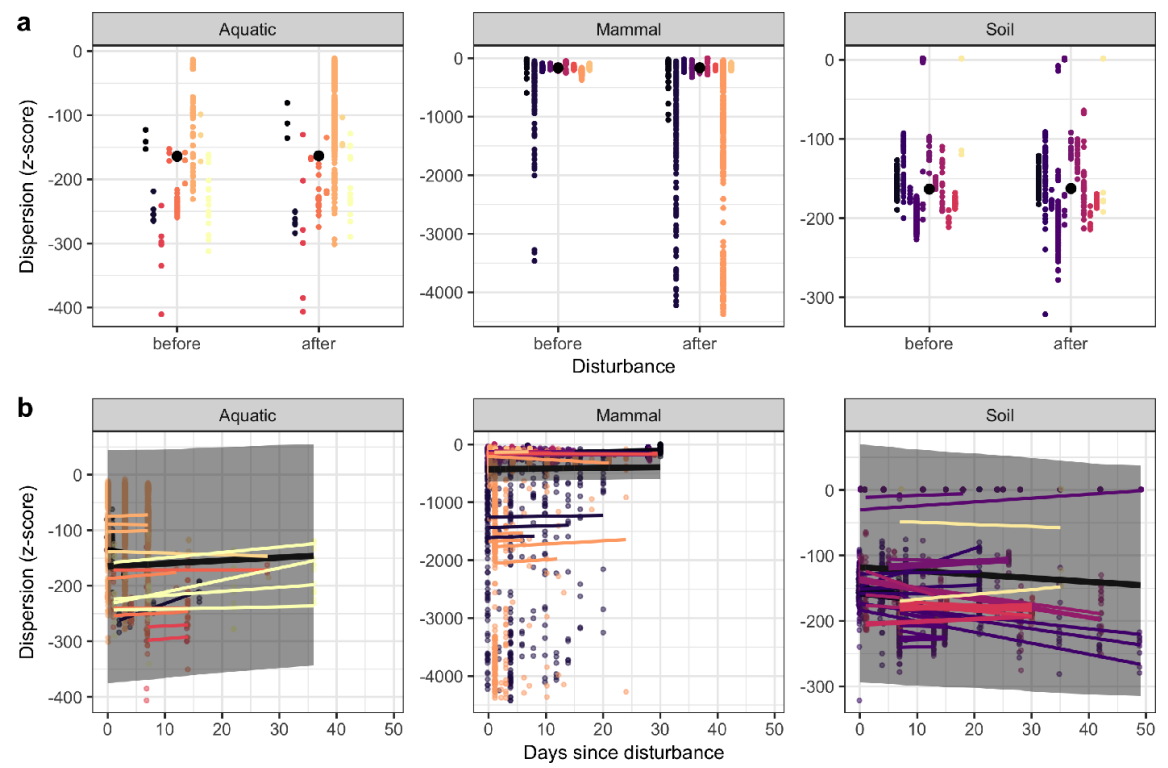

Figure 4. Consistent effect of disturbance on microbiome dispersion are not detected, immediately ( $<4$ days) after disturbance (a), or over 50 days of recovery (b). 

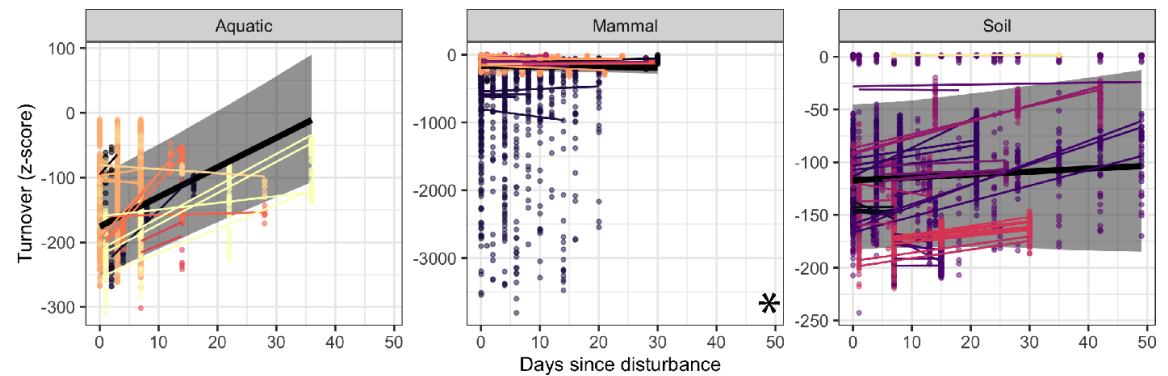

Figure 5. The effect of disturbance on community recovery is environment-dependent.

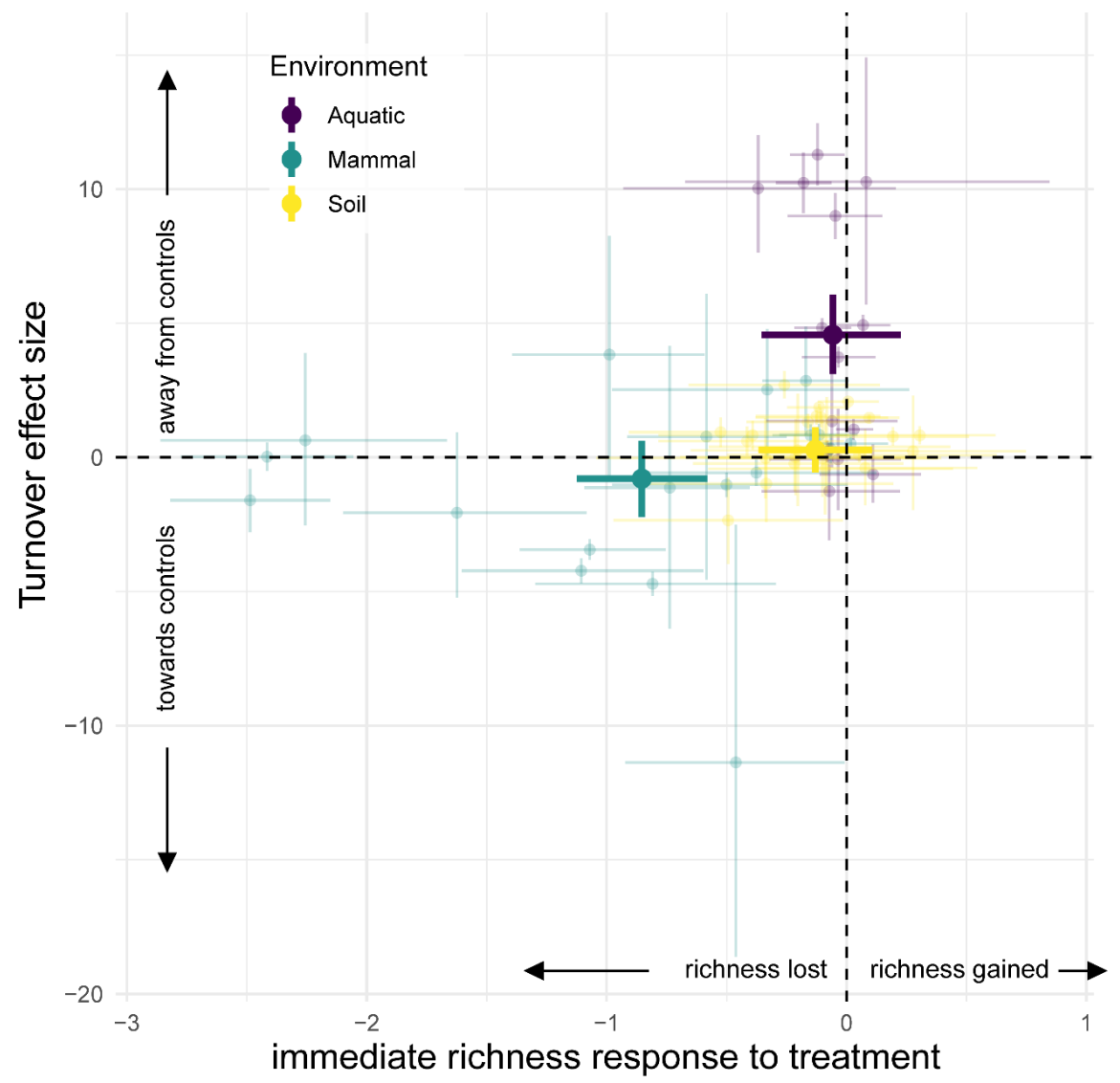

Figure 6. The immediate effect of a disturbance is not related to the microbiome's long-term compositional recovery.

\section{LEGENDS}

Figure 1. Measuring microbial recovery after disturbance. Community richness can be measured before disturbance (yellow), immediately after disturbance (green), and over longer time periods (blue, a). Compositional dynamics can be assessed using compositional dissimilarity metrics (i.e., Bray-Curtis, b). Dispersion can be measured as the pairwise dissimilarity between experimental replicates, and turnover can be measured as the pairwise dissimilarity between pre-disturbance and disturbed communities (red). Following disturbance, communities dispersion can increase or decrease over time if communities become more or less dissimilar, respectively (c). They may also return to their pre-disturbance composition (negative turnover 
trend) or become increasingly dissimilar from the pre-disturbance community (positive turnover trend) Insets are the relationship between time (x-axis) and turnover (dashed line, $\mathrm{y}$-axis) or dispersion (solid line, y-axis).

Figure 2. Samples used in this synthesis. We selected time series that had control samples and multiple samples after disturbance (a). A vertical black line denotes a disturbance event in all cases; samples taken on the day of disturbance (before or after) are shown along this line. A vertical grey line indicates the fourth day after disturbance. Studies which had not sampled the recovering microbiome within $<4$ days after disturbance were excluded from assessments of the immediate impacts of disturbance on richness and dispersion. All samples were standardized to 1,500 observations per sample, and had an average coverage $>0.9$ (b).

Figure 3. The effect of disturbance on microbiome richness, immediately ( $<4$ days) after disturbance (a), and over 50 days of recovery (b). Richness was calculated as the number of observed taxa in each sample, and is presented with a $\log _{2}$-transformed y-axis. Circles represent samples, and are colored by study. In $a$, solid black circles indicate the mean across time series per environment with a $95 \%$ CI indicated by error bars. In $b$, regression lines for each time series are colored by study, and the solid black line shows the mean response across time series per environment. The $95 \%$ CI is displayed as a grey shaded area, and environments for which overall trends deviate from zero are indicated with an asterisk $\left(^{*}\right)$ on the bottom right corner.

Figure 4. Consistent effect of disturbance on microbiome dispersion are not detected, immediately $(<4$ days) after disturbance (a), or over 50 days of recovery (b). Dispersion was calculated as the pairwise Bray-Curtis distance between replicates for each time point within each time series, and each circle is a pairwise comparison, colored by study. In $a$, solid black circles indicate the mean across time series per environment with a $95 \%$ CI. In $b$, regression lines for each time series are colored by study, and a solid black line indicates the mean response across time series per environment. The $95 \%$ CI of the overall response in each environment is displayed as a grey shaded area. y-axis scales differ per environment, and the overall trends from each environment did not deviate from zero.

Figure 5. The effect of disturbance on community recovery is environment-dependent. For each time series, recovery was calculated as the pairwise distance between post-disturbance samples and pre-disturbance controls. Each point is a pairwise comparison, colored by study. recover to increasingly different conformations Regression lines for each time series are colored by study, and a solid black line indicates the mean response across time series per environment. The $95 \%$ CI is displayed as a grey shaded area, and environments for which overall trends deviate from zero are indicated with an asterisk $\left(^{*}\right)$ on the bottom right corner.

Figure 6. The immediate effect of a disturbance is not related to the microbiome's long-term compositional recovery. Each point is a time series, colored by its environment. Immediate richness responses were calculated as the effect of disturbance on community richness (Figure 3a), and log-transformed. Turnover rates were calculated as the slope estimate for turnover over time. Error bars show the $95 \%$ CI for both metrics. Large circles indicate the mean response per environment. 